\title{
LA APLICACIÓN DE HERRAMIENTAS DIGITALES CON EI ENFOQUE ONTOSEMIÓTICO Y SU INFLUENCIA EN EL APRENDIZAJE DE FUNCIONES EXPONENCIALES Y LOGARÍTMICAS
}

\author{
THE APPLICATION OF DIGITAL TOOLS WITH THE ONTOSEMIOTIC APPROACH \\ AND ITS INFLUENCE ON THE LEARNING OF EXPONENTIAL AND LOGARITHMIC \\ FUNCTIONS
}

Referencia del artículo

Ruiz Castillo, J. C. (2022). La aplicación de herramientas digitales con el enfoque ontosemiótico y su influencia en el aprendizaje de funciones exponenciales y logarítmicas. Revista Científica del Sistema de Estudios de Postgrado de la Universidad de San Carlos de Guatemala, 5(1), 15-23. DOI: https://doi.org/10.36958/sep.v5i1.92

\author{
Juan Carlos Ruiz Castillo \\ 2jcruiz@gmail.com \\ https: //orcid.org/0000-0002-2218-1442 \\ Maestría en Ciencia en Didáctica de la Matemática \\ Centro Universitario de Occidente CUNOC
}

\begin{abstract}
RESUMEN
OBJETIVO: determinar la influencia de la aplicación de herramientas digitales con el enfoque ontosemiótico, en el aprendizaje de las funciones exponenciales y logarítmicas en los estudiantes del Profesorado de Enseñanza Media en Ciencias especializado en Física-Matemática, para poder evidenciar los resultados cognitivos. MÉTODO: por el tipo de investigación, el método es descriptivo, porque los resultados obtenidos describieron la situación actual del fenómeno en estudio. RESULTADOS: se determinó los resultados cognitivos al utilizar herramientas digitales haciendo uso del enfoque ontosemiótico, teniendo un efecto significativo en los estudiantes, los cuales presentaron evaluaciones con las siguientes medidas de tendencia central: promedio de 91 puntos, moda de 94 puntos y mediana de 90 puntos; señalando que la aplicación de herramientas digitales más el uso de EOS puede ayudar a incrementar los conocimientos y así mismo el rendimiento académico de los estudiantes. CONCLUSIÓN: se determinó la influencia de la aplicación de herramientas digitales con el Enfoque Ontosemiótico EOS, en el aprendizaje de los estudiantes de Matemática II de la Escuela en Formación de Profesores en Enseñanza Media EFPEM, acerca del contenido de las funciones exponenciales y logarítmicas siendo los resultados obtenidos categorizados como excelentes.
\end{abstract}

\section{PALABRAS CLAVE}

Herramientas digitales, enfoque ontosemiótico, aprendizaje de matemática, funciones exponenciales y logarítmicas

\section{ABSTRACT}

OBJECTIVE: to determine the influence of the application of digital tools with the ontosemiotic approach, in the learning of exponential and logarithmic functions in the students of the High School Teachers in Sciences specialized in Physics-Mathematics, in order to demonstrate the cognitive results. METHOD: due to the type of research, the method is descriptive, because the results obtained described the current situation of the phenomenon under study. RESULTS: the cognitive results were determined when using digital tools using the ontosemiotic approach, having a significant effect on the students, who presented evaluations with the following measures of central tendency: average of 91 points, mode of 94 points and median of 90 points; pointing out that the application of digital tools plus the use of EOS can help increase knowledge and also the academic performance of students. CONCLUSION: the influence of the application of digital tools with the EOS Ontosemiotic Approach was determined, in the learning of the Mathematics II students of the School in Teacher Training in Secondary Education EFPEM, about the content of the exponential and logarithmic functions being the results obtained categorized as excellent.

\section{KEYWORDS}

Digital tools, ontosemiotic approach, mathematics learning, exponential and logarithmic function 


\section{INTRODUCCIÓN}

Fundamentalmente, el aprendizaje de las matemáticas ha sido fuente de muchos estudios, buscando mejorarla, aplicando las diferentes herramientas didácticas, el utilizar técnicas didácticas, provee al estudiante diversas formas de aprender conceptos. Por lo que la presente investigación, evidenciará las herramientas informáticas utilizadas en el aprendizaje de matemática con la aplicación del enfoque ontosemiótico en el Profesorado en Enseñanza Media en Ciencias especializado en Física-Matemática en los estudiantes de la Universidad de San Carlos de Guatemala en la Escuela de Formación de Profesores de Enseñanza Media.

Es importante señalar, que el objetivo de la investigación es determinar la influencia de la aplicación de herramientas digitales con el enfoque ontosemiótico, en el aprendizaje de las funciones exponenciales y logarítmicas en los estudiantes del Profesorado de Enseñanza Media en Ciencias especializado en Física-Matemática, para poder evidenciar los resultados cognitivos.

Dentro del marco metodológico se incluye el método inductivo dirigido a las actividades de: determinación del problema, identificación de la población, selección de la muestra, investigación bibliográfica del marco teórico, diseño del instrumento de recolección de datos, solicitud del permiso correspondiente a los directores del establecimiento. También se hizo uso del método deductivo dirigido a: recopilación de información, tabulando datos, representación de los resultados en gráficas, análisis de datos y obtención de conclusiones para redactar el informe final. La población utilizada en esta investigación fueron los estudiantes de Matemática II del Profesorado en Enseñanza Media especializado en Física-Matemática. Es importante señalar que el estudio es resultado de una tesis de grado de Maestría en Ciencias.

\section{MATERIALES Y MÉTODOS}

Se observó el fenómeno, se realizó la recolección de datos. Se revisaron las notas de pretest y postest y grupo experimental quienes recibieron tratamiento o estímulo experimental. La población de investigación es de 84 estudiantes siendo divididos en dos grupos en el primer grupo se aplicó el experimento, grupo 1 donde participaron 45 estudiantes y grupo 2 donde participaron 39 estudiantes.

\section{RESULTADOS}

A continuación, se presentan los resultados de los conocimientos fundamentales de los pretest de funciones exponenciales y logarítmicas

\section{Grupo 1. Grupo focal al cual se aplicó el experimento}

Con los datos obtenidos en la investigación, se presenta la tabla de frecuencia del pretest del grupo 1, donde se midieron los conocimientos fundamentales que tenían los estudiantes acerca del tema de funciones exponenciales y logarítmicas, esta representa los puntos percentilicos obtenidos en el pretest. 
Tabla 1. Frecuencia del pretest

\begin{tabular}{|c|c|c|c|c|c|c|c|c|c|c|c|c|c|c|}
\hline $\mathrm{Li}$ & Ls & $\mathrm{F}$ & $\mathrm{fa}$ & $x i$ & fxi & $\mathrm{xi}-\bar{x}$ & $|x i-\bar{x}|$ & $f|x i-\bar{x}|$ & $(x i-\bar{x})^{2}$ & $f(x i-\bar{x})^{2}$ & $(x i-\bar{x})^{3}$ & $f(x i-\bar{x})^{3}$ & $(x i-\bar{x})^{4}$ & $f(x i-\bar{x})^{4}$ \\
\hline 0 & 11 & 12 & 12 & 5.5 & 66 & -20.5 & 20.5 & 246 & 420.25 & 5043 & -8615.125 & -103381.5 & 176610.063 & 2119320.75 \\
\hline 12 & 23 & 11 & 23 & 17.5 & 192.5 & -8.5 & 8.5 & 93.5 & 72.25 & 794.75 & -614.125 & -6755.375 & 5220.0625 & 57420.6875 \\
\hline 24 & 35 & 10 & 33 & 29.5 & 295 & 3.5 & 3.5 & 35 & 12.25 & 122.5 & 42.875 & 428.75 & 150.0625 & 1500.625 \\
\hline 36 & 47 & 6 & 39 & 41.5 & 249 & 15.5 & 15.5 & 93 & 240.25 & 1441.5 & 3723.875 & 22343.25 & 57720.0625 & 346320.375 \\
\hline 48 & 59 & 3 & 42 & 53.5 & 160.5 & 27.5 & 27.5 & 82.5 & 756.25 & 2268.75 & 20796.875 & 62390.625 & 571914.063 & 1715742.19 \\
\hline 60 & 71 & 2 & 44 & 65.5 & 131 & 39.5 & 39.5 & 79 & 1560.25 & 3120.5 & 61629.875 & 123259.75 & 2434380.06 & 4868760.13 \\
\hline 72 & 83 & 1 & 45 & 77.5 & 77.5 & 51.5 & 51.5 & 51.5 & 2652.25 & 2652.25 & 136590.875 & 136590.875 & 7034430.06 & 7034430.06 \\
\hline 84 & 95 & 0 & 45 & 89.5 & 0 & 63.5 & 63.5 & 0 & 4032.25 & 0 & 256047.875 & 0 & 16259040.1 & 0 \\
\hline \multirow[t]{2}{*}{96} & 107 & 0 & 45 & 101.5 & 0 & 75.5 & 75.5 & 0 & 5700.25 & 0 & 430368.875 & 0 & 32492850.1 & 0 \\
\hline & & 45 & & & 1171.5 & & & 680.5 & & 15443.25 & & 234876.34 & & 16143494.8 \\
\hline
\end{tabular}

Fuente: propia 2020.

Se puede observar en la tabla de frecuencia señalando que la mayoría de los estudiantes del grupo 1, obtuvo la puntuación de 0 a 11 , también se puede observar, que mientras mayor es el punteo, la cantidad de personas que obtuvieron esa nota es menor. Con los datos obtenidos se puede establecer las medidas de tendencia central: siendo la media aritmética de 26 puntos, la cual señala el promedio de los datos obtenidos; la moda de 11 puntos, la cual indica las notas que más se repitió con mayor frecuencia en los datos adquiridos; y la mediana de 23 puntos, la cual señala la nota que separa la cantidad de estudiantes que obtuvieron notas altas y bajas.

Es transcendental señalar, que las medidas de tendencia central, resumen el comportamiento de los datos, representando un centro en torno al cual se localiza el conjunto de datos. Con los datos adquiridos, es incuestionable que se tenía un desconocimiento del tema de funciones exponenciales y logarítmicas, pues las puntuaciones que señalan las medidas de tendencia central, tienen datos percentilicos muy bajos.

También se pudo establecer las medidas de dispersión siendo la desviación media de 15 , la varianza de 343, la desviación típica de 19 y un coeficiente de variación de $71 \%$. Se puede evidenciar una asimetría positiva, siendo el dato de 0.82 con el coeficiente de Fisher, y una curtosis de 3.04 señalando una agudeza leptocúrtica.

\section{Grupo 2. No se aplicó el experimento}

En los datos que se obtuvieron del pretest, se midieron los conocimientos fundamentales que poseían los estudiantes acerca del tema de funciones exponenciales y logarítmicas, esta representa los puntos percentilicos obtenidos en el pretest. 
Se obtuvieron los siguientes datos en las medidas de tendencia central, siendo la media aritmética de 36 puntos, la cual señala el promedio de los datos obtenidos; la moda de 51 puntos, la cual indica las notas que más se repitieron con mayor frecuencia en los datos adquiridos y la mediana de 30 puntos, la cual señala la nota que separa la cantidad de estudiantes que obtuvieron notas altas y bajas relativas.

Con los datos adquiridos, se pudo establecer que tuvieron un desconocimiento parcial del tema de funciones exponenciales y logarítmicas. Empero, es importante señalar, que los punteos son mejores que los obtenidos con el grupo 1 , las puntuaciones que señalan las medidas de tendencia central tienen datos percentilicos bajos.

Los cálculos para las medidas de dispersión son los siguientes: la desviación con respecto a la media que es 17 , esta magnitud señala la variación que se tienen respecto a la media, la varianza 390 señalando la dispersión que representa la variabilidad de una cantidad de datos a su media. La desviación estándar indica que tan dispersos están los datos con respecto a la media en el pretest del grupo 2 es de 20 .
De igual manera, es importante señalar al coeficiente de variación, hace referencia a la relación del tamaño de media y la variabilidad de la variable siendo en está tabla un $57 \%$.

También se puede evidenciar una asimetría negativa con el coeficiente de Fisher siendo el dato de -0.11 , la cual es catalogada como negativa, lo cual significa que la distribución de las notas está colocada en la derecha indicando que estos datos en su mayoría están comprendidos en los intervalos regulares y bajos, y una curtosis de 1.68 siendo evidente la agudeza platicúrtica. Las medidas de dispersión señalan cuanto se alejan los datos respecto a la media.

\section{Resultados del postest del tema funcio- nes exponenciales y logarítmicas}

Se presenta la tabla de frecuencia del postest del grupo 1, donde se midieron los conocimientos que obtuvieron los estudiantes después de recibir las orientaciones, es importante destacar que en este grupo se aplicó el experimento haciendo uso de herramientas virtuales con el uso del EOS, en los temas de funciones exponenciales y logarítmicas.

\section{Tabla 2. Frecuencia del postest}

\begin{tabular}{|c|c|c|c|c|c|c|c|c|c|c|c|c|c|c|}
\hline $\mathrm{Li}$ & Ls & $\mathrm{F}$ & $\mathrm{fa}$ & $x i$ & $f x i$ & $\mathrm{xi}-\bar{x}$ & $|x \mathbf{i}-\bar{x}|$ & $f|x i-\bar{x}|$ & $(x i-\bar{x})^{2}$ & $f(x i-\bar{x})^{2}$ & $(x i-\bar{x})^{3}$ & $f(x i-\bar{x})^{3}$ & $(x i-\bar{x})^{4}$ & $f(x i-\bar{x})^{4}$ \\
\hline 70 & 74 & 1 & 1 & 72 & 72 & -19 & 19 & 19 & 361 & 361 & -6859 & -6859 & 130321 & 130321 \\
\hline 75 & 79 & 0 & 1 & 77 & 0 & -14 & 14 & 0 & 196 & 0 & -2744 & 0 & 38416 & 0 \\
\hline 80 & 84 & 9 & 10 & 82 & 738 & -9 & 9 & 81 & 81 & 729 & -729 & -6561 & 6561 & 59049 \\
\hline 85 & 89 & 3 & 13 & 87 & 261 & -4 & 4 & 12 & 16 & 48 & -64 & -192 & 256 & 768 \\
\hline 90 & 94 & 17 & 30 & 92 & 1564 & 1 & 1 & 17 & 1 & 17 & 1 & 17 & 1 & 17 \\
\hline \multirow[t]{2}{*}{95} & 100 & 15 & 45 & 97 & 1455 & 6 & 6 & 90 & 36 & 540 & 216 & 3240 & 1296 & 19440 \\
\hline & & 45 & & & 4090 & & & 219 & & 1695 & & 10355 & & 209595 \\
\hline
\end{tabular}

Fuente: propia 2020. 
Se puede observar en la tabla frecuencia del primer grupo, teniendo un incremento de los punteos después de aplicar el experimento, como se puede evidenciar, las notas correspondientes a 90 a 94 fueron las que más se repitieron, mientras que 8 estudiantes obtuvieron los punteos de 80 a 84 y mientras que un estudiante obtuvo el punteo de 70 a 74 . Con los datos adquiridos, es indudable la diferencia cognitiva con relación al pretest, pues en el pretest que se tenía un desconocimiento del tema, ahora con el postest se tiene un conocimiento más sólido en cuanto al contenido. Es significativo indicar, que las puntuaciones que señalan las medidas de tendencia central tienen datos percentilicos muy altos.

Con relación a lo anterior, las medidas de tendencia central, siendo la media aritmética de 91 puntos, la cual señala el promedio de los datos obtenidos; la moda de 94 puntos, la cual señala las notas que más se repitieron y la mediana 90 puntos, la cual señala la nota que separa los más altos y los más bajos. También, se pudo establecer las medidas de dispersión siendo la desviación media de 5 , la varianza de 38, la desviación estándar de 6 y un coeficiente de variación de $7 \%$.

Es trascendental señalar, que estas medidas permiten el conocer la característica de su asimetría y su homogeneidad, en esta tabla se obtuvo un coeficiente de asimetría de Fisher de -0.99, la cual es catalogada como negativa, que significa que la distribución de las notas está colocada en la derecha indicando que estos datos en su mayoría están comprendidos en los intervalos excelentes, siendo evidentes en la figura 2 y una curtosis de 1.68 el termino leptocúrtica señala que la curva tiene una agudeza muy alta.

Figura 1. Comparación de pretest y postest

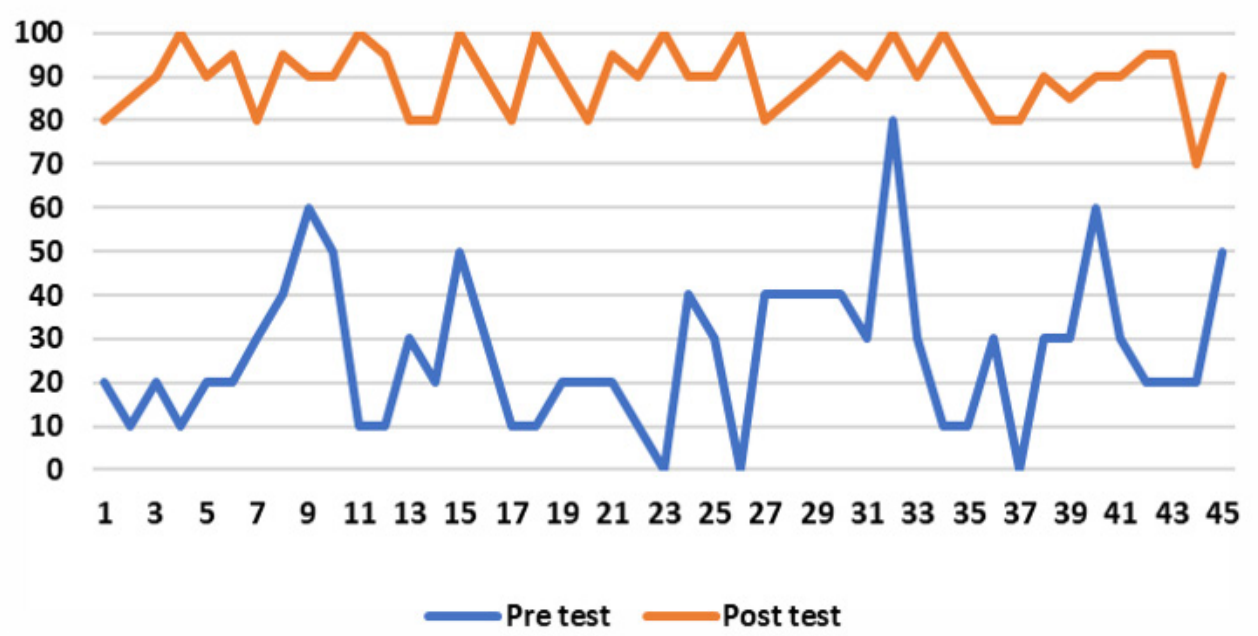

Fuente: propia 2020. 
En la Figura 1, se evidencia el logro cognitivo que obtuvieron los estudiantes al ser capacitados con el enfoque ontosemiótico y herramientas virtuales en la enseñanza de las funciones exponenciales y logarítmicas, siendo la gráfica azul la que señala la nota en percentil obtenida en el pretest y la gráfica en naranja, señala la nota en percentil obtenida en el postest, esto indica evidentemente la diferencia que obtuvo cada estudiante, siendo la nota más baja en el postest de 70 puntos.

Con los datos adquiridos, es evidente la diferencia cognitiva con relación al pretest, pues en el mismo se asevera que se tenía un desconocimiento del tema de funciones exponenciales y logarítmicas, ahora con el postest se tiene un conocimiento más sólido en cuanto al tema. Es significativo indicar, que las puntuaciones que señalan las medidas de tendencia central tienen datos percentilicos muy altos.

Con relación a lo anterior, las medidas de tendencia central, siendo la media aritmética de 91 puntos, la cual señala el promedio de los datos obtenidos; la moda de 94 puntos, la cual señala las notas que más se repitieron y la mediana 90 puntos, la cual señala la nota que separa los más altos y los más bajos. También, se pudo establecer las medidas de dispersión siendo la desviación media de 5 , la varianza de 38, la desviación estándar de 6 y un coeficiente de variación de $7 \%$.

En cuanto a la desviación con respecto a la media que es 5 , esta magnitud señala la variación que se tienen respecto a la media, la varianza 38 señala la dispersión que representa la variabilidad de una cantidad de datos a su media. La desviación estándar de 6 indica que tan dispersos están los datos con respecto a la media.

De igual manera, es trascendental indicar al coeficiente de variación, que hace referencia a la relación del tamaño de media y la variabilidad de la variable siendo en esta tabla un $7 \%$.
En cuanto a la asimetría y curtosis brindan información sobre la distribución de una variable. Es importante resaltar, que estas medidas permiten el conocer la característica de su asimetría y su homogeneidad, en esta tabla se obtuvo un coeficiente de asimetría de -0.99 , la cual es catalogada como negativa, lo cual significa que la distribución de las notas está colocada en la derecha indicando que estos datos en su mayoría están comprendidos en los intervalos excelentes, siendo evidentes en los resultados y una curtosis de 1.68 el termino leptocúrtica señala que la curva tiene una agudeza muy alta con relación a la media.

\section{Grupo 2}

Para el grupo 2 se puede comparar el logro cognitivo que obtuvieron los estudiantes al ser capacitados de forma tradicional en la enseñanza de las funciones exponenciales y logarítmicas. Se obtuvo un logro en algunos estudiantes, sin embargo, a comparación del grupo 1 estos logros no son tan altos, inclusive se puede señalar que algunos de los estudiantes no superaron la nota que obtuvieron en el pretest.

En los resultados del postest del grupo 2 obtuvo un mayor punteo en las notas correspondientes a 33 a 41 , una cantidad de menor de estudiantes obtuvo los punteos de 60 a 68 y otra aún menor a la anterior obtuvo notas de 78 a 86. Con los datos obtenidos, se puede establecer las medidas de tendencia central, siendo la media aritmética de 56 puntos, la cual señala el promedio de los datos obtenidos; la moda de 94 puntos, la cual indica las notas que más se repitieron con mayor frecuencia en los datos adquiridos y la mediana 39 puntos, la cual señala la nota que separa la cantidad de estudiantes que obtuvieron notas altas y bajas. Es importante señalar, que las medidas de tendencia central resumen el comportamiento de los datos, 
representando un centro en torno al cual se localiza el conjunto de datos.

Es evidente la diferencia cognitiva con relación al pretest, pues en el pretest que se tenía un desconocimiento del tema, ahora con el postest se tiene un conocimiento bueno en cuanto al tema. Con relación a lo anterior, se pudo establecer las medidas de dispersión siendo la desviación media de 14, la varianza de 283, la desviación estándar de 17 y un coeficiente de variación de $30 \%$.

Se puede observar, el incremento con relación al pretest sin embargo no están grande como al grupo que se le aplicó el experimento.

En cuanto a la desviación, con respecto a la media que es 14 , la varianza 283. La desviación estándar indica que tan dispersos los datos con respecto a la media, la cual es de 17. El coeficiente de variación es de un $30 \%$. Se puede evidenciar una asimetría positiva siendo el dato de 0.48 , y una curtosis de 1.99 siendo evidente la agudeza platicúrtica.

\section{La correlación del pretest y el postest en el grupo experimental}

Como lo menciona Mendehal, Beaver y Beaver citado por Ruiz (2017), “El coeficiente de correlación como una medida de la fuerza de relación lineal entre dos variables" (p.44).

La correlación que se obtuvo entre el pretest y postest teniendo un coeficiente de correlación -0.81 siendo está considerada como correlación negativa aceptable. Con la correlación negativa se puede interpretar lo siguiente, si la nota de los evaluados en el pretest es menor, la nota en el postest será mayor.

Según Rodas (2002) señala que: el cociente de correlación lo podemos interpretar de acuerdo con los siguientes casos:
Si r es positivo, la correlación entre las variables es positiva

Si $r$ es negativo, la correlación entre las variables es negativa

Si $r=0$, no existe relación lineal entre las variables

Si $r=1$, la correlación positiva es perfecta

Si $r=-1$, la correlación negativa es perfecta

Si $0.90<r<1$ o $-1<r<-0.90$ la correlación es excelente

Si $0.80<r<0.90$ o $-0.90<r<-0.80$, la correlación es aceptable

Si $0.60<r<0.80$ o $-0.80<r<-0.60$ la correlación es regular

Si $0.30<r<0.60$ o $-0.60<r<-0.30$ la correlación es mínima

Si $0<r<0.30$ o $-0.30<r<0$ no hay correlación (p.10)

\section{DISCUSIÓN}

Se determinó los resultados cognitivos al utilizar herramientas digitales haciendo uso del enfoque ontosemiótico, teniendo un efecto significativo en los estudiantes, pues presentaron evaluaciones con las siguientes medidas de tendencia central: promedio de 91 puntos, moda de 94 puntos y mediana de 90 puntos; señalando que la aplicación de herramientas digitales más el uso de EOS puede ayudar a incrementar los conocimientos y así mismo el rendimiento académico de los estudiantes. Por otra parte, las herramientas que los estudiantes consideraron como ideales para la enseñanza de las funciones exponenciales y logarítmicas son: mimio, GeoGebra y graphmatica, señalando la influencia que también genera el uso de herramientas virtuales. 
Resulta evidente que los estudiantes piensan y procesan la información de modo significativamente distinto a sus predecesores. Además, no es un hábito coyuntural, sino que está llamado a prolongarse en el tiempo, que no se interrumpe, sino que se acrecienta, de modo que su destreza en el manejo y utilización de la tecnología es superior a la de sus profesores y educadores (Prensky, 2010, p. 5).

Al correlacionar el aprendizaje de funciones exponenciales y logarítmicas con herramientas digitales haciendo uso del EOS, con una enseñanza tradicionalista aplicando entornos virtuales, se obtuvo una correlación de -0.81 siendo catalogada como correlación negativa excelente demostrando que los resultados del pretest fueron superados en el postest, al aplicar las herramientas virtuales y el Enfoque Ontosemiótico optimizando el aprendizaje y el rendimiento académico de los estudiantes.

\section{REFERENCIAS}

Prensky, M. (2010). Nativos e imigrantes digitales. SEK, S.A. obtenido por https:// marcprensky.com/writing/Prensky-NA-
TIVOS\%20E\%2OINMIGRANTES\%20DIGITALES\%20(SEK).pdf

Rodas, I. (2002). Estadística. Guatemala. Zantamaró. Ediciones S. A.

Ruiz, J (2017) "La aplicación de las herramientas didácticas en el aprendizaje de la Matemática. Estudio realizado en la Licenciatura en la enseñanza de la matemática y la física de la EFPEM-USAC". Obtenido por http://biblioteca.usac. edu.gt/tesis/29/29_0540.pdf

\section{SOBRE EL AUTOR}

\section{Juan Carlos Ruiz Castillo}

Es profesor en la Enseñanza Media Especializado en Física-Matemática, Licenciado en la Enseñanza de la Matemática y la Física, Maestría en Ciencias en Formación Docente, Maestría en Ciencias en Didáctica de la Matemática todas las carreras estudiadas en la Universidad de San Carlos de Guatemala, ha publicado un artículo en la Revista de Etnomatemática "Los factores sociales asociados a los modelos matemáticos en un contexto de pandemia".

Conflicto de intereses

Declara no tener ningún conflicto de intereses.

Declaración de consentimiento informado

El estudio se realizó, respetando el Código de ética y buenas prácticas editoriales de publicación.

Derechos de uso

Copyright@ 2022 por Juan Carlos Ruiz Castillo

Este texto está protegido por una licencia Creative Commons 4.0. Internacional

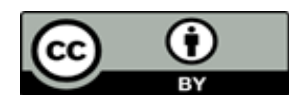

Usted es libre para compartir, copiar y redistribuir el material en cualquier medio o formato y adaptar el documento, remezclar, transformar y crear a partir del material para cualquier propósito, incluso comercialmente, siempre que cumpla la condición de atribución: usted debe reconocer el crédito de una obra de manera adecuada, proporcionar un enlace a la licencia, e indicar si se han realizado cambios. Puede hacerlo en cualquier forma razonable, pero no de forma tal que sugiera que tiene el apoyo del licenciante o lo recibe por el uso que hace. 